\title{
Der Schlußparagraph des Buches vom Atmen, das Isis machte
}

\author{
Joachim Friedrich Quack
}

\section{Summary}

The final section of the "Book of Breathings which Isis made" has posed serious problems for previous translators. A new translation is proposed here, taking into consideration the relatively late state of the language of the section. The text prescribes that the mummy should be formed with the hands clasped above the heart on the breast, and the funerary document wrapped in textile, placed below the left arm and included in the outer wrappings.

Das „Buch vom Atmen, das Isis machte" ist inzwischen in seinen Umrissen einigermaßen bekannt geworden und vom „ersten “ und „zweiten Buch vom Atmen " unterschieden worden. ${ }^{1}$ Dennoch gibt es einzelne Probleme der Übersetzung. Hier will ich mich auf den Schlußabschnitt ${ }^{2}$ konzentrieren, der wohl am schwierigsten ist und in bisherigen Übersetzungen auch noch öfters mit Fragezeichen und unübersetzten Passagen versehen ist. ${ }^{3}$ Tatsächlich dürfte sich allerdings der größte Teil der Schwierigkeiten unmittelbar lösen, sobald man erkennt, daß hier neuägyptische bzw. teilweise bereits demotische Sprachelemente vorhanden sind. Der betreffende Abschnitt ist derjenige, welcher die

1 Vgl. besonders M. Coenen, J. Quaegebeur, De papyrus Denon in het Museum MeermannoWestreenianum, Den Haag of Het Boek van het ademen van Isis (Leuven 1995); M. Coenen, An Introduction to the Document of Breathing made by Isis, RdÉ 49 (1998), 37-45;ders., The Funerary Papyri of the Bodleian Library at Oxford, JEA 86 (2000), 81-98, dort 87-96; ders., Owners of Documents of Breathing Made by Isis, CdÉ 79 (2004), 59-72; Fr.-R. Herbin, Trois manuscrits originaux du Livre des respirations fait par Isis (P. Louvre N 3121, N. 3083 et N 3166), RdÉ 50 (1999), 149-239; ders., Catalogue of the Books of the Dead and Other Religious Texts in the British Museum, Volume IV. Books of Breathing and Related Texts (London 2008), 1-2 u. 11-49; M.D. Rhodes, The Hor Book of Breathings. A Translation and Commentary (Provo 2002). Auch die alte Publikation von P. J. de Horrack, Oeuvres Le livre des respirations (Partis 1877), wieder abgedruckt in P. J. de Horrack, Euvres divers, Bibliothèque égyptologique 17 (Paris 1907), 109-137, Taf. VII-XIII, ist für einige Punkte noch wichtig. Erst nach Abschluß dieses Manuskripts erschien M. Smith, Traversing Eternity. Texts for the Afterlife from Ptolemaic and Roman Egypt (Oxford 2009), wo 467 u. 475f. eine mit meiner Interpretation quasi identische Auffassung geboten wird. Da Smith seine von früheren Bearbeitern teilweise abweichende Übersetzung nicht mit philologischen Anmerkungen begründet hat, scheint mir dieser Aufsatz nicht überflüssig geworden zu sein.

2 Im Papyrus des Hor (pJoseph Smith I+XI+X) steht der Abschnitt nicht am Schluß, sondern am Anfang der Handschrift.

3 Ich berücksichtige im Kommentar die Übersetzungen von de Horrack, Euvres divers, 135f.; J.-C. Goyon, Rituels funéraires de l'ancien Égypte (Paris 1972), 228f.; Herbin, RdÉ 50, 167; Rhodes, Hor Book of Breathings, 27f. 
praktische Durchführung des Rituals angibt; in den meisten Handschriften wird er ausgelassen. Ansonsten wird er gerne im Layout als eigene Sektion markiert, so beginnt pLouvre N 3121, 7, 11 mit ihm eine neue Zeile (das Ende der vorangehenden wird freigelassen). pLouvre N 3284 und pJoseph Smith setzen ihn für sich auf eine Seite.

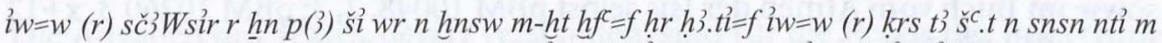

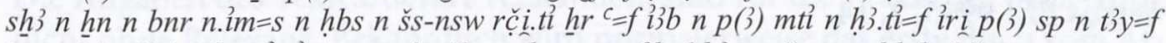

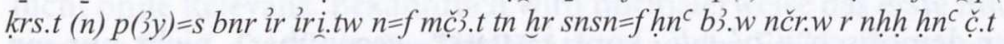

„Man soll ${ }^{\mathrm{a}}$ ) Osiris hereinbringen zum großen Becken des Chons, nachdem er über seinem Herzen eine Faust geballt hat. ${ }^{b}$ ) Man soll das Dokument zum Atmen, das innen und außen an sich beschriftet ist, in Stoff aus Byssos einwickeln, ${ }^{c}$ ) wobei es unter seinen linken Arm gelegt wird ${ }^{d)}$ gegenüber ${ }^{\mathrm{e}}$ ) seinem Herzen. Man soll den $\operatorname{Rest}^{\mathrm{f}}$ ) seiner Wicklung außen um es machen $^{g}$ ). Wenn man dieses Buch für ihn macht, dann atmet er mit den Seelen der Götter in alle Ewigkeit.“

\section{Philologische Anmerkungen}

a) Syntaktisch kann hier nur ein Futur III vorliegen, nicht das von de Horrack („on remorque l'Osiris“) und Herbin („On hale Osiris“) offenbar angenommene und von Rhodes mit der Umschrift $i w=w\langle h r\rangle$ st. ${ }^{3}$ explizit angesetzte Präsens, das st (hrr) sč̉ lauten würde (sowohl im Neuägyptischen als auch im Demotischen). Auch Goyons Deutung als Umstandssatz "quand on hale“ ist syntaktisch wenig plausibel, insbesondere, da er das an sich ganz gleichartige $i w=w(r) k r s$ dann als Hauptsatz „on procède“ auffaßt.

b) Das Verb $h f^{c}$ wird nicht mit der Präposition $h r$ konstruiert, folglich sind die Übersetzungen von de Horrrack („après qu'il a repris son cœur“), Goyon („,et qu'il a repris possession de son cœur") und Herbin („après qu'il a saisi son cœur") nicht akzeptabel. Vielmehr ist $h f^{c}$ intransitiv als „die Faust ballen“ $\mathrm{zu}$ verstehen. Vgl. dazu im Balsamierungsritual pBoulaq III, 7, 7 wti.hr $\mathrm{p}=t w$ çr.t=f ỉb nti hft "dann bandagiert man seine linke Hand, die zur Faust geballt ist". ${ }^{4}$ pJoseph Smith hat hier ausführlicher formuliert $m$ - ht $[h f]^{c}{ }^{c}$. wi $=f 2 r$ $h 3 . t i=f$,nachdem seine beiden Arme eine Faust geballt haben in Richtung zum Herzen“; vgl. die Übersetzung von Rhodes (,after his two hands have been clasped to his heart").

c) krs bedeutet nicht global „bestatten“, sondern spezifischer „in Stoff einwickeln“. ${ }^{5}$ Nur so ist auch der Eintrag zum Zeichen (bekanntlich einem Stab

4 S. Sauneron, Rituel de l'embaumement (Kairo 1952), 24.

5 Vgl. in diesem Sinne bereits J. F. Quack, Die rituelle Erneuerung der Osirisfigurinen, WdO 31 (2000/01), 5-18, dort S. 13 Anm. 32. 
mit darum gewickeltem Stoff) im Zeichenpapyrus von Tanis zu verstehen, der [...] $i w=f k r s,[\ldots]$, das in Stoff gewickelt ist" lautet. ${ }^{6}$

d) So im pJoseph Smith XI und pLouvre N 3284. pLouvre N 3121 hat iri.ti. Wechsel von $i r i$ und $r \grave{c} i$ ist mir auch sonst im römerzeitlichen Hieratisch bekannt, so u.a. in einigen unpublizierten Passagen des Buches vom Tempel sowie im Buch vom Atmen der Isis selbst pBM 10048, 7, 3; pBM 10260 3, x+12 versus BM 9995, 3, 14.

e) Vgl. hier demotisch $n$ p3 $m t i$, kopt. M̄тmHт „gegenüber", nicht nur ein vages ,à proximité de“, wie Herbin übersetzt. pJoseph Smith bietet stattdessen einfaches $m t i$.

f) Goyon und Rhodes fassen das Zeichen $\Longrightarrow$ als rmn "Träger" auf. Zur Lesung als $s p$ vgl. J.F. Quack, Fragmente memphitischer Religion und Astronomie in semidemotischer Schrift (pBerlin $14402+$ pCarlsberg $651+$ PSI Inv. D 23), in: F. Hoffmann, H. J. Thissen (Hrsg.), Res severa verum gaudium. Festschrift für Karl-Theodor Zauzich zum 65. Geburtstag am 8. Juni 2004, Studia Demotica 6 (Leuven/Paris/Dudley, MA 2004), 467-496, T. 37-39, dort $473 \mathrm{Anm}$. k; sie wird für den vorliegenden Fall durch die demotische Notiz im pLouvre N 3291 gestützt (s.u.), wo die Gruppe für $s p$ unmöglich als $r m n$ interpretiert werden kann. pJoseph Smith ist die Lesung $t^{3} y=f$ eindeutig; ebenso pLouvre N 3121. pLouvre N 3126 ist die Gruppe beschädigt, der Abstrich des $f$ jedoch in de Horracks Faksimile einigermaßen klar. pLouvre N 3284 könnte nach de Horracks Faksimile da, wo man $t$ i $y=f$ erwartet, verderbt sein; (⿻日木 111

die bisherige Lesung 1 III ist jedenfalls paläographisch problematisch. Auch inhaltlich wäre die Erwähnung der Position der Sargträger im Vergleich zu den sonstigen Angaben wenig kongruent.

g) Herbin läßt diesen Passus unübersetzt; Goyon versteht „Les neuf Porteurs procèdent à l'ensevelissement en laissant le verso [apparent] "; Rhodes übersetzt "while the bearer of his coffin works on its outside“. Die Form des pLouvre 3284 läßt sich als Infinitiv zur Handlungsanweisung verstehen. Dagegen ist Doo e

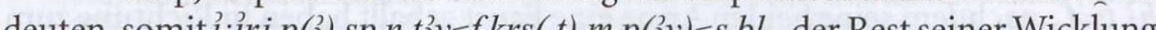
deuten, somit $i: \operatorname{ir} i p(3) s p n t^{3} y=f k r s(. t) m p(3 y)=s b l$,der Rest seiner Wicklung ist außen um es" zu übersetzen, mit einem autofokalen zweiten Tempus, wie es

6 F. Ll. Griffith, W.M. F. Petrie, Two Hieroglyphic Papyri from Tanis (London 1889), 16, Taf. IIIIV, Kol. XV, 2 (ich gehe davon aus, daß am Anfang der Erklärung ein Wort verloren ist, da die Kolumne mit der Deutung andernfalls im Vergleich zu sonstigen im Papyrus auffällig schmal wäre). Vgl. auch E. Hornung, Der Eine und die Vielen (Darmstadt 1971), 22f. mit einer zu direkten Deutung auf die Bestattung. 
im Demotischen hinreichend belegt ist. ${ }^{7}$ pLouvre 3284 hat nach de Horracks Faksimile $p 3 y=s$ in $p 3 y$ verderbt.

\section{Inhaltlicher Kommentar}

Die Angaben des Textes, derart verstanden, sind für die Praxis der Beisetzung nicht ohne Relevanz. Bekanntlich wird normalerweise das erste Buch vom Atmen am Kopf, das zweite Buch vom Atmen an den Füßen der Mumie plaziert. ${ }^{8}$ Die hier vorliegende Anweisung gibt für das „Buch vom Atmen, das Isis für ihren Bruder Osiris gemacht hat" eine noch andere Position an, nämlich unter dem linken Arm auf Höhe des Herzens, wobei es in den Prozeß der Wicklung integriert wird. Relevant ist auch die Angabe zur Armposition. ${ }^{9}$ Die Mumie soll so arrangiert werden, daß der (rechte) Arm mit einer geballten Faust über dem Herzen positioniert ist - also so, wie es tatsächlich normale Praxis bei männlichen Mumien der 18.-21. Dynastie ist. ${ }^{10}$ Allerdings kommt diese Haltung entgegen den Angaben älterer Standardwerke auch zu anderen Zeiten vor, so im Mittleren Reich ${ }^{11}$ und der Spätzeit sowie griechisch-römischen Zeit, ${ }^{12}$ so daß man mit Rückschlüssen auf die konkrete Entstehungszeit des Textes vorsichtig sein sollte. ${ }^{13}$

Weiter ausgebaut werden kann diese Interpretation durch einen demotischen Vermerk auf dem Verso des pLouvre N 3291. ${ }^{14}$ Dabei handelt es sich um einen weiteren Textzeugen für das Buch vom Atmen, das Isis gemacht hat. In ihm ist der hieratische Text des oben diskutierten Paragraphen nicht präsent, wohl aber eine sachlich korrespondierende Notiz auf der Rückseite

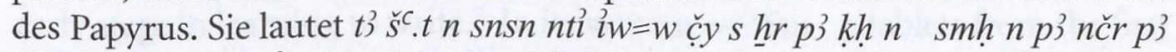

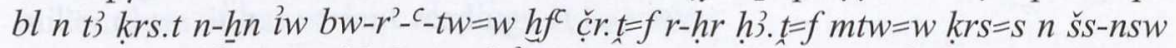

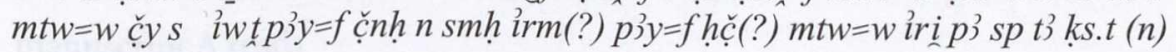

7 J.F. Quack, Zu Syntax und Zeitbezug der demotischen zweiten Tempora, LingAeg 14 (2006), 251-262, bes. 259 .

8 Herbin, Books of Breathing, 2-3.

9 Für die folgenden Bemerkungen danke ich Beatrix Geßler für ihre fachkundige Unterstützung. Vgl. P.H. Gray, Notes concerning the Position of Arms and Hands of Mummies with a View to possible Dating, JEA 58 (1972), 200-204.

10 G. Elliot Smith, W.R. Dawson, Egyptian Mummies (London 1924), 91, $102 f$.

11 Mumie des $W 3 h$, S. Ikram, A. Dodson, The Mummy in Ancient Egypt. Equipping the Dead for Eternity (London 1998), 159.

12 Ikram, Dodson, The Mummy, 129f.; M. Raven, W. Taconis, Egyptian Mummies. Radiological Atlas of the Collections in the National Museum of Antiquities at Leiden (Turnhout 2005), 39.

13 Sprachlich ist der Text sicher relativ jung, wobei der letzte Satz mit seinem ir iri.tw allerdings als vordemotisch einzustufen ist.

14 De Horrack, Oeuvres divers, Taf. XIII; E. Revillout, Une famille de paraschistes ou taricheutes thébains, ZÄS 17 (1879), 83-92, dort 92, Taf. VI (Nr. 23) (mit Nennung der Nummer als 2891). 
p3 $y=s[b l] t 3 s^{c} y . t n$ snsn r:iri 3 s.t $n$ Wsir t3i „Das Buch vom Atmen, das man unter den linken Arm des Gottes legen soll außerhalb der inneren Wicklung, bevor man seine Hand zur Faust ballt, hin zu seinem Herzen, und man soll es in Byssos wickeln, und man soll es zwischen seinen linken Unterarm und seine Achsel ${ }^{15}$ geben, und man soll den Rest der Wicklung [außen] um es machen. Das ist das Buch vom Atmen, das Isis für Osiris machte". Bei andersartiger Formulierung der Details stimmt dieser Text in der Sache vollständig überein.

15 Das Wort ist anhand der verfügbaren Faksimiles von de Horrack und Revillout mit einiger

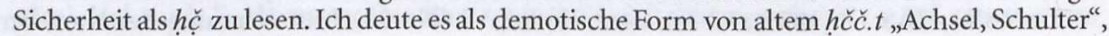
koptisch $x O, x \omega$, a $\sigma \omega$. 